\title{
Prevalence of Comorbid Anxiety Disorders and Their Associated Factors in Patients with Bipolar Disorder or Major Depressive Disorder
}

This article was published in the following Dove Press journal: Neuropsychiatric Disease and Treatment

\author{
Takeshi Inoue $\mathbb{D}^{\prime}$ \\ Toshifumi Kimura $\mathbb{D D}^{2}$ \\ Yoshifumi Inagaki ${ }^{2}$ \\ Osamu Shirakawa ${ }^{3}$ \\ 'Department of Psychiatry, Tokyo \\ Medical University, Tokyo, Japan; ${ }^{2}$ Medical \\ Affairs Department, Medical Division, \\ GlaxoSmithKline K.K., Tokyo, Japan; \\ ${ }^{3}$ Department of Neuropsychiatry, Kindai \\ University Faculty of Medicine, Osaka, \\ Japan
}

\begin{abstract}
Objective: Comorbid anxiety disorders in patients with mood disorders have a negative impact on outcomes, such as persistence of depressive symptoms, deterioration of quality of life (QoL), increased suicide risk, mood instability with antidepressant treatment, but often go underrecognized in clinical practice. To identify features useful for supporting the confirmation of comorbid anxiety disorders, we investigated the prevalence of comorbid anxiety disorders and their associated factors in Japanese patients with mood disorders using data from our previously reported JET-LMBP study.
\end{abstract}

Patients and Methods: Patients with bipolar disorder (BD; $n=114)$ and patients with major depressive disorder (MDD; $n=334$ ), all with major depressive episodes (DSM-IV-TR) were analyzed. Comorbid anxiety disorders were confirmed using the Mini-International Neuropsychiatric Interview. Demographic and clinical features were assessed using patient background forms, including the Quick Inventory of Depressive Symptomatology-Self Report Japanese version, 36-Item Short-Form Health Survey (SF-36), and Child Abuse and Trauma Scale (CATS). Multivariate logistic regression analysis adjusted for age, sex, and severity of depressive symptoms was used to identify factors associated with comorbid anxiety disorders (post hoc analysis).

Results: The prevalence of comorbid anxiety disorders was significantly higher in patients with $\mathrm{BD}(53.2 \%)$ than in patients with MDD (37.2\%). Factors associated with comorbid anxiety disorders in BD included no spouse, interpersonal rejection sensitivity, higher CATS sexual abuse scores, and lower SF-36 mental component summary scores. In MDD, factors included hypersomnia, pathological guilt feelings, higher CATS neglect scores, and lower SF-36 physical component summary scores.

Conclusion: Comorbid anxiety disorders were commonly seen in Japanese patients with mood disorders. Childhood abuse, atypical depression symptoms, and deterioration of healthrelated QoL were commonly associated with comorbid anxiety disorders in BD and MDD, suggesting that the presence of these features may be useful to support the confirmation of comorbid anxiety disorders in these patients.

Keywords: anxiety disorders, bipolar disorder, comorbidity, major depressive disorder, prevalence

\section{Introduction}

Patients with mood disorders have a variety of comorbid psychiatric disorders, including anxiety disorders and substance use disorders. ${ }^{1,2}$ The Sequenced Treatment Alternatives to Relieve Depression (STAR*D) trial, a large-scale study conducted in outpatients with major depressive disorder (MDD), found that $65.2 \%$
Department of Psychiatry, Tokyo Medical University, 6-7-I Nishishinjuku, ShinjukuKu, Tokyo 160-0023, Japan

Tel +81333426111

Fax +8I $33345 \quad 1437$

Email tinoue@tokyo-med.ac.jp 
of patients had at least one psychiatric disorder. ${ }^{3}$ Among comorbid psychiatric disorders, anxiety disorders were commonly seen $(11.8-31.3 \%){ }^{3}$ In the Systematic Treatment Enhancement Program for Bipolar Disorder (STEP-BD), a large-scale study of outpatients with bipolar disorder (BD), anxiety disorder was the most commonly observed comorbidity $(30.5 \%) .{ }^{4}$ This high rate of comorbidity may reflect the direct or indirect influence of a primary psychiatric disorder on the development of a subsequent disorder, and the existence of a common cause between both disorders through genetic predisposition and socioeconomic factors. ${ }^{5}$ Anxiety disorders commonly develop in early life, but the risk of comorbidity between anxiety disorders and MDD has been shown to remain at consistently high levels across age groups. ${ }^{6}$

Comorbid anxiety disorders are known to negatively affect mood disorder outcomes. A prospective study of the long-term course of depression demonstrated that patients with comorbid anxiety disorders had increased persistence of depression, required more time for depressive episode remission, and had more disability than those without comorbid anxiety disorders. ${ }^{7,8}$ It was also shown that patients with BD and comorbid anxiety disorders required more time to recover from depressive symptoms, experienced earlier relapse, and had lower quality of life (QoL) than those without comorbid anxiety disorders. ${ }^{9}$ The risk of suicide is a significant concern in mood disorders. A meta-analysis of several previous studies indicated that comorbid anxiety disorder was a risk factor for suicide and suicide attempts in patients with depressive disorder or $\mathrm{BD},{ }^{10,11}$ suggesting that comorbid anxiety disorders have a potential to increase suicide risk in patients with mood disorders. It is well known that patients with $\mathrm{BD}$ and anxiety disorders exhibit impairments in a variety of cognitive domains, such as attention and executive functions. ${ }^{12,13}$ Therefore, cognitive impairment may cause the negative impact of comorbid anxiety disorders on psychosocial functions, such as disability, QoL and suicidality in patients with mood disorders. However, the contribution of comorbid anxiety disorders to worsening impairment of psychosocial functioning could be negligible, given that cognitive impairment in patients with both $\mathrm{BD}$ and obsessive-compulsive disorder (OCD) did not worsen more than in those with either BD or OCD. ${ }^{14}$ Comorbid anxiety disorders are also known to have a negative impact on the effect of antidepressants, which are often prescribed for mood disorders. According to an earlier report, comorbid anxiety disorders were significantly associated with treatment resistance to antidepressants. ${ }^{15}$ Furthermore, it is likely that comorbid anxiety disorders are associated with mood lability with antidepressants. In earlier studies, patients with mood disorder and comorbid anxiety disorders reported antidepressant-induced mood switches to hypomania/mania more often than those without comorbid anxiety disorders. ${ }^{16,17}$

Considering the negative impact of comorbid anxiety disorders on treatment and outcomes in patients with mood disorder, it is important to check for comorbid anxiety disorders and provide careful treatment if anxiety disorders are detected. However, comorbid anxiety disorders in patients with depression or BD are often overlooked and ignored in routine clinical practice. ${ }^{18,19}$ In fact, the prevalence of comorbid anxiety disorders diagnosed by a routine clinical interview (23.6\%) was more than two times lower than by a semistructured interview $(57.3 \%)$ in patients with a primary diagnosis of MDD. ${ }^{19}$ This discrepancy in prevalence rates between the two interview styles indicates that it is challenging to fully recognize anxiety symptoms unless a patient specifically raises concerns, as it is difficult to incorporate the use of timeconsuming semi-structured interviews into routine clinical practice. Furthermore, it has been noted that the diagnosis of comorbid anxiety disorders is complicated due to the following: 1) obsession, worries, obsessiveness, and physical symptoms associated with comorbid anxiety disorders may be masked by irritability and rapid mood changes, which are particularly evident in young patients with $\mathrm{BD}$, and 2) patients tend to ignore anxiety symptoms and complain about BDrelated behavioral problems. ${ }^{20}$ Considering these problems, identifying the features associated with comorbid anxiety disorders in patients with mood disorder may be useful in routine clinical practice. Physicians may consider these features when identifying potential patients with comorbid anxiety disorders, and when carefully determining the presence or absence of anxiety disorders.

Several studies have examined the factors associated with comorbid anxiety disorders in patients with mood disorders. A mental health survey conducted in the general population in the Netherlands revealed that, in patients with mood disorder, the following characteristics were associated with comorbid anxiety disorder: female gender, younger age, lower educational level, and unemployment. ${ }^{21}$ Two other studies demonstrated that early onset of $\mathrm{BD}$, family history of substance use disorder, severity of depressive symptoms, and use of antidepressants were associated with comorbid anxiety disorders in both in- and outpatients with BD. ${ }^{22,23}$ However, to our knowledge, no study has compared the factors associated with comorbid anxiety disorders between 
patients with $\mathrm{BD}$ and MDD. In our previous report, a Japanese epidemiological trial with the latest measure of BD (the JET-LMBP study), we obtained comprehensive information on various demographic and clinical features (including the presence or absence of current anxiety disorders) in BD and MDD outpatients with major depressive episodes (MDEs). ${ }^{24}$ Thus, using data from the JET-LMBP study (post hoc analysis), we investigated the prevalence of comorbid anxiety disorders and their associated factors in outpatients with mood disorders presenting with MDE.

\section{Patients and Methods \\ Patients and Assessment}

The JET-LMBP study was a multicenter, cross-sectional, and epidemiological study conducted from April to June 2013 in 448 outpatients aged between 20 and 65 years who had MDE based on the Diagnostic and Statistical Manual of Mental Disorders, 4th Edition, Text Revision (DSM-IV-TR).

The study was completed at 23 psychiatric institutions (Supplementary Text). The JET-LMBP study was conducted in accordance with the Declaration of Helsinki and the Japanese Ethical Guidelines for Epidemiological Research. Before beginning the study, the protocol was reviewed and approved by the institutional review board or ethics committee of each facility. Before any study procedures were performed, written informed consent was obtained from all patients. To protect the personal information of patients, a unique and study-specific subject identification number was assigned to each patient for linkable anonymity.

After informed consent was obtained, eligible patients were interviewed using the Mini-International Neuropsychiatric Interview (M.I.N.I) to determine the presence of mood episodes and psychiatric comorbidities such as anxiety disorder and substance-related disorder. ${ }^{25,26}$ Mood disorders were diagnosed using DSMIV-TR based on information obtained and psychiatrists' examination. Anxiety disorders included panic disorder (PD), agoraphobia, social anxiety disorder (SAD), OCD, post-traumatic stress disorder (PTSD), and generalized anxiety disorder (GAD). In addition, various demographic and clinical characteristics were assessed by physicians using a patient background form. The form consisted of 52 questions, including basic patient demographic and clinical information such as age, sex, family history, age of onset number of episodes, and current symptoms such as mood reactivity, hypersomnia, and excessive eating.

In addition, patients were asked to complete three self-reported questionnaires: the Quick Inventory of Depressive Symptomatology-Self Report Japanese version (QIDS-J), to assess the severity of depressive symptoms for the previous 7 days (according to MDD diagnostic criteria [based on DSM-IV]); ${ }^{27}$ the Japanese version of the 36-item Short-form Health Survey (SF$36)$, to assess health-related QoL (HRQoL); ${ }^{28}$ and the Japanese version of the 38-item Child Abuse and Trauma Scale (CATS), to assess how frequently a particular abusive experience occurred during the patient's childhood and adolescence. ${ }^{29,31}$ QIDS-J is composed of 16 items (score from 0 to 3), which cover the nine DSM-IV criterion diagnostic symptom domains: 1) sad mood (1 item), 2) concentration (1 item), 3) self-criticism (1 item), 4) suicidal ideation (1 item), 5) interest (1 item), 6) energy/fatigue (1 item), 7) sleep disturbance (initial, middle, and late insomnia or hypersomnia) (4 items), 8) decrease or increase in appetite or weight (4 items) and 9) psychomotor agitation or retardation ( 2 items). The total score ranges from 0 (normal) to 27 (very severe); the maximum score is selected as a representative value among items for domain 7), 8), and 9). SF-36 consists of eight subscales: physical functioning, role-physical, bodily pain, general health perception, vitality, social functioning, roleemotional, and mental health. Each subscale provides a standardized score (from 0 to 100), with lower scores indicating poorer health or higher disability. Scores on the first four subscales and the latter four subscales are used to calculate physical component summary scores and mental component summary scores, respectively. Both variables were computed according to the formulas provided in the SF-36 user's manual and were expressed as standard scores. CATS is a scale that assigns scores from 0 (never) to 4 (always), to 38 types of abuse experiences. Total and Neglect/Sexual abuse/ Punishment scores were represented as mean scores from all items and relevant items, respectively.

\section{Statistical Analysis}

SAS System 9.3 (SAS Institute Inc., Cary, NC, USA) was used for analysis. All statistical analyses were performed using a two-sided significance level of $<0.05$. Fisher's exact test or the two-sample $t$-test was used to examine the comparison between the two groups, 
stratified by the presence or absence of comorbid anxiety disorders in terms of demographic characteristics. Fisher's exact test was used to check the comparison in the proportion of comorbid anxiety disorders between BD and MDD.

To identify factors associated with comorbid anxiety disorders, univariate logistic regression analyses were performed for the demographic and clinical features of BD or MDD patients with/without comorbid anxiety disorders. For features with a $P$-value $<0.1$ in the univariate logistic regression analyses, except the CATS total score and eight SF-36 subscales, multivariate logistic regression analyses adjusted for age, sex, and QIDS-J were performed. The stepwise method (entering $=0.05$, removing $=0.05$ ) was used to choose factors. To avoid multicollinearity, only one-factor pair was chosen if highly correlated factor pairs were included, considering the phi coefficient or Spearman's rank correlation coefficient. Finally, the predictive performance of the multivariate logistic regression equation was evaluated by a receiver-operating characteristic curve analysis.

\section{Results}

\section{Patient Characteristics}

Of 114 patients with BD, 109 had information on the presence or absence of each anxiety disorder, and 58 (53.2\%) had comorbid anxiety disorders. Of 334 patients with MDD, 317 had information on the presence or absence of each anxiety disorder, and 118 (37.2\%) had comorbid anxiety disorders. The prevalence of comorbid anxiety disorders in patients with $\mathrm{BD}$ was significantly higher than that in patients with MDD $(P<0.005)$ (Table 1). Furthermore, the frequency of each comorbid anxiety disorder tended to be higher in patients with BD than patients with MDD.

The demographic characteristics of patients with BD, with comorbid anxiety disorders $(n=58)$ and without comorbid anxiety disorders, $(n=51)$ are presented in Table 2 . The

Table I Prevalence of Comorbid Anxiety Disorders in Patients with BD and MDD with Major Depressive Episodes

\begin{tabular}{|l|l|l|l|l|l|}
\hline \multirow{2}{*}{} & \multicolumn{2}{l|}{ BD } & \multicolumn{2}{l|}{ MDD } & *P-value \\
\cline { 2 - 6 } & N & \% (n) & N & \% (n) \\
\hline Any anxiety disorder & 109 & $53.2(58)$ & 317 & $37.2(118)$ & 0.005 \\
Panic disorder & 114 & $8.8(10)$ & 330 & $8.2(27)$ & 0.845 \\
Agoraphobia & 107 & $16.8(18)$ & 314 & $11.1(35)$ & 0.131 \\
Social anxiety disorder & 114 & $29.8(34)$ & 333 & $16.2(54)$ & 0.003 \\
Obsessive-compulsive disorder & 114 & $6.1(7)$ & 331 & $3.0(10)$ & 0.157 \\
Post-traumatic stress disorder & 114 & $3.5(4)$ & 332 & $2.4(8)$ & 0.513 \\
Generalized anxiety disorder & 114 & $14.0(16)$ & 332 & $10.2(34)$ & 0.302 \\
\hline
\end{tabular}

Notes: *Fisher's exact test was used.

Abbreviations: BD, bipolar disorder; MDD, major depressive disorder.

Table 2 Demographic Characteristics of Patients with BD Stratified by the Presence or Absence of Comorbid Anxiety Disorders

\begin{tabular}{|l|l|l|l|}
\hline Characteristics & Comorbid Anxiety Group(N=58*) & Non-Comorbid Anxiety Group(N=5 I*) & P-value \\
\hline Age (years), mean \pm SD & $36.2 \pm 9.0$ & $39.5 \pm 11.5$ & $0.090^{\mathrm{a}}$ \\
Sex (male), N (\%) & $23(39.7)$ & $22(43.1)$ & $0.846^{\mathrm{b}}$ \\
History of marriage, N (\%) & $27(47.4)$ & $33(64.7)$ & $0.083^{\mathrm{b}}$ \\
History of divorce, N (\%) & $12(20.7)$ & $12(23.5)$ & $0.818^{\mathrm{b}}$ \\
History of spouse's death, N (\%) & $3(5.2)$ & $1(2.0)$ & $0.621^{\mathrm{b}}$ \\
Presence of spouse, N (\%) & $15(25.9)$ & $25(49.0)$ & $0.017^{\mathrm{b}}$ \\
Presence of child, N (\%) & $17(29.3)$ & $26(51.0)$ & $0.030^{\mathrm{b}}$ \\
Presence of housemate, N (\%) & $41(70.7)$ & $43(84.3)$ & $0.112^{\mathrm{b}}$ \\
Duration of education (years), mean \pm SD & $13.5 \pm 2.7$ & $14.0 \pm 2.2$ & $0.333^{\mathrm{a}}$ \\
Currently employed, N (\%) & $22(37.9)$ & $25(49.0)$ & $0.253^{\mathrm{b}}$ \\
History of job turnover, N (\%) & $40(69.0)$ & $30(60.0)$ & $0.419^{\mathrm{b}}$ \\
\hline
\end{tabular}

Notes: *Subject with missing data was excluded. $P$-values were calculated using Two-sample. $t$-test ${ }^{\mathrm{a}}$ or Fisher's exact test ${ }^{\mathrm{b}}$.

Abbreviations: BD, bipolar disorder; SD, standard deviation. 
Table 3 Demographic Characteristics of Patients with MDD Stratified by the Presence or Absence of Comorbid Anxiety Disorders

\begin{tabular}{|l|l|l|l|}
\hline Characteristics & Comorbid Anxiety Group(N=I I 8*) & Non-Comorbid Anxiety Group(N= I98*) & $P$ value \\
\hline Age (years), mean \pm SD & $40.0 \pm 9.1$ & $39.9 \pm 11.0$ & $0.918^{\mathrm{a}}$ \\
Sex (male), N (\%) & $43(36.4)$ & $88(44.4)$ & $0.194^{\mathrm{b}}$ \\
History of marriage, N (\%) & $65(55.1)$ & $98(49.7)$ & $0.415^{\mathrm{b}}$ \\
History of divorce, N (\%) & $29(24.8)$ & $32(16.2)$ & $0.076^{\mathrm{b}}$ \\
History of spouse's death, N (\%) & $1(0.9)$ & $7(3.5)$ & $0.266^{\mathrm{b}}$ \\
Presence of spouse, N (\%) & $41(34.7)$ & $67(33.8)$ & $0.903^{\mathrm{b}}$ \\
Presence of child, N (\%) & $55(46.6)$ & $83(41.9)$ & $0.482^{\mathrm{b}}$ \\
Presence of housemate, N (\%) & $79(66.9)$ & $147(74.6)$ & $0.156^{\mathrm{b}}$ \\
Duration of education (years), mean \pm SD & $12.9 \pm 2.5$ & $13.8 \pm 2.2$ & $0.002^{\mathrm{a}}$ \\
Currently employed, N (\%) & $50(42.4)$ & $118(59.6)$ & $0.004^{\mathrm{b}}$ \\
History of job turnover, N (\%) & $82(69.5)$ & $112(57.4)$ & $0.04 I^{\mathrm{b}}$ \\
\hline
\end{tabular}

Notes: *Subject with missing data was excluded. P-values were calculated using Two-sample. $t$-test ${ }^{\mathrm{a}}$ or Fisher's exact test ${ }^{\mathrm{b}}$.

Abbreviations: MDD, major depressive disorder; SD, standard deviation.

patients with $\mathrm{BD}$ and comorbid anxiety disorders were less likely to have a spouse and child than those without comorbid anxiety disorders. The demographic characteristics of patients with MDD with $(\mathrm{n}=118)$ and without comorbid anxiety disorders $(\mathrm{n}=199)$ are presented in Table 3 . The patients with MDD and comorbid anxiety disorders showed a lower number of completed years of education, a lower frequency of current employment, and a greater history of job turnover compared with those without comorbid anxiety disorders.

\section{Identification of Factors Associated with Comorbid Anxiety Disorders in Patients with BD}

Univariate logistic regression analysis was performed for 73 features after patients with BD were classified into those with and without comorbid anxiety disorders (Supplementary Table 1). Features with $P$-values $<0.1$ in the univariate logistic regression analysis were identified as candidate factors associated with comorbid anxiety disorders. The total CATS score and eight subscales of SF-36 were excluded from the candidate factors associated with comorbid anxiety disorders for multivariate logistic regression analysis. This was to avoid multicollinearity between the total CATS score and three subscales (CATS sexual abuse score, CATS neglect score, and CATS punishment score), as well as the eight SF-36 subscales and the twocomponent summary scores. Multivariate logistic regression analysis adjusted for age, sex, and QIDS-J showed that patients without spouses and those with interpersonal rejection sensitivity were 6.6 and 3.5 times more likely to have comorbid anxiety disorders, respectively (Table 4).
Higher CATS sexual abuse scores and lower SF-36 mental component summary scores were also associated with a higher likelihood of comorbid anxiety disorders. When the cutoff value was 0.5 , sensitivity, specificity, accuracy, and area under the curve (AUC) were $73.6 \%, 55.3 \%$, $65.0 \%$, and 0.800 , respectively.

\section{Identification of Factors Associated with Comorbid Anxiety Disorders in Patients with MDD}

Univariate logistic regression analysis was also performed for 73 features after patients with MDD were classified into those with or without comorbid anxiety disorders (Supplementary Table 2). The features included in the multivariate logistic regression analysis were selected

Table 4 Multivariate Logistic Regression Analysis of Features Correlated with Comorbid Anxiety Disorders in Patients with $\mathrm{BD}$ and Major Depressive Episodes

\begin{tabular}{|l|l|l|l|}
\hline Features & P-value & $\begin{array}{l}\text { Odds } \\
\text { Ratio }\end{array}$ & $\mathbf{9 5 \%} \mathbf{~ C l}$ \\
\hline Age & 0.4759 & 1.018 & $0.969-1.07 I$ \\
Sex (Male) & 0.9800 & 1.013 & $0.38 I-2.690$ \\
QIDS-J score & 0.5897 & 1.033 & $0.919-1.161$ \\
Presence of spouse & 0.0016 & 0.151 & $0.047-0.488$ \\
Interpersonal rejection & 0.0104 & 3.541 & $1.346-9.316$ \\
sensitivity & & & \\
SF-36: Mental component & 0.0139 & 0.923 & $0.866-0.984$ \\
summary & & & \\
CATS: Sexual abuse score & 0.0186 & 6.818 & $1.378-33.740$ \\
\hline
\end{tabular}

Abbreviations: $\mathrm{BD}$, bipolar disorder; $\mathrm{Cl}$, confidence interval; CATS, Child Abuse and Trauma Scale; QIDS-J, Quick Inventory of Depressive Symptomatology-Self Report, Japanese version; SF-36, 36-item Short-form Health Survey. 
Table 5 Multivariate Logistic Regression Analysis of Features Correlated with Comorbid Anxiety Disorders in Patients with MDD and Major Depressive Episodes

\begin{tabular}{|l|l|l|l|}
\hline Features & P-value & $\begin{array}{l}\text { Odds } \\
\text { Ratio }\end{array}$ & $\mathbf{9 5 \%} \mathbf{C l}$ \\
\hline Age & 0.5117 & 0.984 & $0.937-1.033$ \\
Sex (Male) & 0.2470 & 1.836 & $0.656-5.137$ \\
QIDS-J score & 0.3960 & 0.950 & $0.843-1.070$ \\
Hypersomnia & 0.0168 & 7.417 & $1.435-38.34 \mathrm{I}$ \\
Pathological guilt feelings & 0.0003 & 8.038 & $2.567-25.167$ \\
SF-36: Physical component & 0.0231 & 0.943 & $0.897-0.992$ \\
summary & & & \\
CATS: Neglect score & 0.0254 & 2.051 & $1.092-3.852$ \\
\hline
\end{tabular}

Abbreviations: CATS, Child Abuse and Trauma Scale; $\mathrm{Cl}$, confidence interval; MDD, major depressive disorder; QIDS-J, Quick Inventory of Depressive SymptomatologySelf Report, Japanese version; SF-36, 36-item Short-form Health Survey.

using a similar method to that used for patients with BD. Multivariate logistic regression analysis, adjusting for age, sex, and QIDS-J, showed that patients with hypersomnia and those with pathological guilty feelings were 8 and 7.4 times more likely to have comorbid anxiety disorders, respectively (Table 5). Higher CATS neglect scores and lower SF-36 physical component summary scores were also more likely to be associated with comorbid anxiety disorders. When the cutoff value was 0.5 , sensitivity, specificity, accuracy, and AUC were 58.5\%, 86.5\%, 76.5\%, and 0.827 , respectively.

\section{Discussion}

In this study, the prevalence of comorbid anxiety disorders in outpatients with BD and MDE (53.2\%) was significantly higher than in outpatients with MDD (37.2\%). The STAR*D study showed that the prevalence of comorbid anxiety disorder in outpatients with MDD was $42.3 \%$ and the prevalence of SAD was the highest among comorbid anxiety disorders, ${ }^{3,32}$ which was similar to the results of this study. However, the prevalence of comorbid anxiety disorders in outpatients with BD in the STEP-BD study $(30.5 \%)$ was notably lower than in this study. ${ }^{33}$ In a metaanalysis of 30 previous studies in $\mathrm{BD}$, prevalence was $38.2 \%,{ }^{34}$ which was also lower than in our study. The advantage of our study seems to be the diagnosis of comorbid anxiety disorders in both patients with BD and patients with MDD under the same conditions among participating psychiatric institutions. However, since our results are derived from one study in Japan, further studies in a larger sample size from various countries are needed to determine whether a difference in the prevalence of comorbid anxiety disorders exists between BD and MDD.

In this study, factors associated with comorbid anxiety disorders in $\mathrm{BD}$ were the absence of a spouse, interpersonal rejection sensitivity, higher CATS sexual abuse scores, and lower SF-36 mental component summary scores. In contrast, factors associated with comorbid anxiety disorders in MDD were hypersomnia, pathological guilty feelings, higher CATS neglect scores, and lower SF-36 physical component summary scores. Interestingly, we found three common features associated with comorbid anxiety disorders between BD and MDD: history of childhood abuse (higher sexual abuse and neglect scores), presence of atypical depression symptoms (interpersonal rejection sensitivity and hypersomnia), and reduced HRQoL (lower mental and physical component summary scores).

Several studies have supported the association between childhood abuse and comorbid anxiety disorders in mood disorders. ${ }^{35,37}$ One study examined which type of childhood abuse (emotional neglect, psychological abuse, physical abuse, sexual abuse) was associated with two-year subsequent comorbid MDD and anxiety disorders in participants who had neither MDD or anxiety disorders at baseline. Consistent with our results, emotional neglect was the only risk factor for subsequent comorbidity with both disorders. ${ }^{38}$ The other group also reported that women with both MDD and anxiety disorders reported more emotional neglect and emotional abuse experiences than those with either MDD or anxiety disorder alone. ${ }^{39}$

In contrast, the contribution of each type of childhood abuse to comorbid anxiety disorders in patients with $\mathrm{BD}$ is inconsistent among previous studies. Two studies indicated an association of sexual abuse with comorbid anxiety disorder, which supports the results of this study. One study examining the effects of physical abuse and sexual abuse on the course of BD demonstrated that patients with a history of either, or both, abuses were more likely to have comorbid anxiety disorders. ${ }^{40}$ Additionally, a meta-analysis showed that sexual abuse had a significant association with future development of anxiety disorders. ${ }^{41}$ On the other hand, Pavlova's research group demonstrated that emotional abuse and physical abuse were significantly associated with comorbid anxiety disorders in patients with $\mathrm{BD},{ }^{35}$ which was inconsistent with the results of our study. Pavlova's research group used the Childhood Trauma Questionnaire (CTQ) as the child abuse assessment scale, which differed from the assessments used in our study. In our study, the total CATS 
score, including questions regarding emotional abuse and physical abuse, tended to be associated with comorbid anxiety disorders in the univariate logistic regression analysis (Supplementary Table 1). Further investigation on the contribution of each type of abuse to comorbid anxiety disorders in patients with $\mathrm{BD}$ is required.

We found that symptoms of atypical depression (interpersonal rejection sensitivity in $\mathrm{BD}$ and hypersomnia in MDD) are factors associated with comorbid anxiety disorders, which is supported by previous studies. The prevalence of comorbid SAD or PD in atypical depression, identified by the presence of hypersomnia and hyperphagia, was significantly higher than in depression without atypical features, suggesting that hypersomnia may be associated with comorbid anxiety disorders in MDD. ${ }^{42}$ It was reported that there was a difference in the pattern of atypical symptoms between bipolar and unipolar depressions. $^{43}$ Compared with unipolar depression, patients with bipolar depression demonstrated more interpersonal rejection sensitivity. Similarly, Benazzi's report showed that the proportion of patients with interpersonal rejection sensitivity was significantly higher in patients with bipolar II disorder than those with MDD. ${ }^{44}$ Furthermore, a meta-analysis examining the relationship of interpersonal rejection sensitivity with mental disorders showed an association with depression or anxiety. ${ }^{45}$ Considering these findings, interpersonal rejection sensitivity may be identified as an associated factor with comorbid anxiety disorders in BD in this study.

In $\mathrm{BD}$, the absence of a spouse was found to be associated with comorbid anxiety disorders. In a nationally representative community mental health survey in Canada, people without spouses were around 1.4 to 1.5 times more likely to have mood disorders with MDEs or manic episodes and comorbid anxiety disorders than those with spouses. ${ }^{46}$ The World Health Organization World Mental Health survey found that marriage was associated with a lower risk of developing BD or anxiety disorders in both sexes, and a lower risk of developing MDD in men. ${ }^{47}$ These results appear to support our finding that the absence of a spouse was associated with comorbid anxiety disorders in BD only.

In contrast, pathological guilt feelings, one of the core depression symptoms, were found to be associated with comorbid anxiety disorders only in MDD. Although there are few reports on the association between pathological guilt feelings and anxiety disorders, the NIMH-ECA survey in the general US population indicated that patients with comorbid
MDD and PD were 1.8 times more likely to have pathological guilt feelings than those with MDD alone. ${ }^{48}$

In this study, deterioration of HRQoL (lower SF-36 mental component summary scores in BD and lower SF36 physical component summary scores in MDD) was identified as a factor associated with comorbid anxiety disorders. It has been reported that the severity of depressive symptoms is correlated with an impaired QoL in patients with mood disorder. ${ }^{49,51}$ Thus, in this study, a multivariate logistic regression model adjusted for depressive symptom severity was used to clarify the association between QoL, independent of the severity of depressive symptoms and comorbid anxiety disorders. Using a multivariate logistic regression model adjusted for depressive symptom severity, a previous study revealed that lower mental health scores and lower physical health scores were associated with current comorbid anxiety disorders in patients with BD, and only lower mental health scores were associated with lifetime comorbid anxiety disorders, ${ }^{52}$ which supported our study findings. One study identified that lower physical health scores were associated with anxious depression in patients with MDD, ${ }^{53}$ which was also consistent with the results of this study.

There are some limitations to this study. Firstly, in the identification of factors associated with comorbid anxiety disorders, OCD and PTSD were classified as anxiety disorders according to DSM-IV. However, currently, they are categorized by DSM-5 as separate from anxiety disorders such as PD and SAD, based on possible differences in the etiology and pathogenesis of these disorders. Approximately $8-10 \%$ of patients with comorbid anxiety disorders also had OCD or PTSD. Since multivariate analysis stratified by each disorder was not feasible due to the small number of patients with each comorbidity, further investigation is needed to address whether there is a difference in factors associated with the comorbidity between OCD or PTSD and other anxiety disorders. Secondly, childhood abuse was retrospectively evaluated by asking patients to recall childhood experiences, which may be influenced by recollection bias. Thirdly, the severity of depression may be overestimated or underestimated because it was assessed by the QIDS-J, which is a self-administered rating scale, rather than physician ratings, such as the Hamilton Depression Rating Scale and MontgomeryÅsberg Depression Rating Score. 
Finally, there are major points to be investigated in future studies. This study identified features associated with comorbid anxiety disorders in patients with a pre-existing confirmed diagnosis of anxiety disorders using M.I.N.I. The predictive ability of comorbid anxiety disorders using these features in mood disorder patients with undiagnosed anxiety disorders needs to be confirmed by a prospective study. Childhood abuse and deterioration of HRQoL associated with comorbid anxiety disorders in this study were assessed using a timeconsuming rating scale. In order to apply these features to routine clinical practice more widely, the development of methods to assess them more easily would be essential.

\section{Conclusion}

In Japanese outpatients with MDE, the prevalence of comorbid anxiety disorders in $\mathrm{BD}$ was $53.2 \%$, which was significantly higher than in MDD (37.2\%). The present study identified three common features associated with comorbid anxiety disorders between BD and MDD: childhood abuse, atypical depression symptoms, and deterioration of HRQoL. These features were supported by previous reports. Although further studies to confirm the predictive ability of comorbid anxiety disorders are needed, the presence of these features may be useful in supporting the confirmation of comorbid anxiety disorders in patients with mood disorders.

\section{Abbreviations}

AUC, area under the curve; BD, bipolar disorder; CATS, Child Abuse and Trauma Scale; CI, confidence interval; DSM-IV-TR, Diagnostic and Statistical Manual of Mental Disorders, 4th Edition, Text Revision; GAD, generalized anxiety disorder; GAF, Global Assessment of Functioning; HRQoL, health-related quality of life; MDD, major depressive disorder; MDE, major depressive episode; M.I.N.I, Mini-International Neuropsychiatric Interview; n.c., not calculated; OCD, obsessive-compulsive disorder; PD, panic disorder; PTSD, post-traumatic stress disorder; QIDS-J, Quick Inventory of Depressive Symptomatology-Self Report Japanese version; QoL, quality of life; SAD, social anxiety disorder; SD, standard deviation; SF-36, 36-Item Short-Form Health Survey.

\section{Acknowledgments}

Statistical analysis was conducted by CMIC PMS.

\section{Funding}

This study (Study No. 117285) was funded by GlaxoSmithKline.

\section{Disclosure}

Takeshi Inoue has received personal fees from GlaxoSmithKline, Mochida Pharmaceutical, Asahi Kasei Pharma, and Shionogi; grants from Astellas; and grants and personal fees from Otsuka Pharmaceutical, Dainippon Sumitomo Pharma, Eli Lilly, Eisai, Mitsubishi Tanabe Pharma, Pfizer, AbbVie GK, MSD, Yoshitomiyakuhin, Takeda Pharmaceutical, and Meiji Seika Pharma; and is a member of the advisory boards of GlaxoSmithKline, Pfizer, Eli Lilly, Mochida Pharmaceutical and Mitsubishi Tanabe Pharma. Osamu Shirakawa has received honoraria from Eli Lilly; Otsuka Pharmaceutical; Kyowa Pharmaceutical; GlaxoSmithKline. Yoshifumi Inagaki, who is stockholder of GlaxoSmithKline, and Toshifumi Kimura are full-time employees of GlaxoSmithKline. The authors report no other conflicts of interest in this work.

\section{References}

1. Bauer M, Pfennig A, Severus E, et al. World federation of societies of biological psychiatry (WFSBP) guidelines for biological treatment of unipolar depressive disorders, part 1: update 2013 on the acute and continuation treatment of unipolar depressive disorders. World J Biol Psychiatry. 2013;14(5):334-385. doi:10.3109/15622975.2013.804195

2. Vieta E, Berk M, Schulze TG, et al. Bipolar disorders. Nat Rev Dis Primers. 2018;4(1):18008. doi:10.1038/nrdp.2018.8

3. Trivedi MH, Rush AJ, Wisniewski SR, et al. Evaluation of outcomes with citalopram for depression using measurement-based care in STAR*D: implications for clinical practice. Am J Psychiatry. 2006;163(1):28-40. doi:10.1176/appi.ajp.163.1.28

4. Bowden CL, Perlis RH, Thase ME, et al. Aims and results of the NIMH systematic treatment enhancement program for bipolar disorder (STEP-BD). CNS Neurosci Ther. 2012;18(3):243-249. doi:10.1111/j.1755-5949.2011.00257.x

5. Lai HM, Cleary M, Sitharthan T, Hunt GE. Prevalence of comorbid substance use, anxiety and mood disorders in epidemiological surveys, 1990-2014: a systematic review and meta-analysis. Drug Alcohol Depend. 2015;154:1-13. doi:10.1016/j.drugalcdep.2015.05.031

6. Essau CA, Lewinsohn PM, Lim JX, Ho MR, Rohde P. Incidence, recurrence and comorbidity of anxiety disorders in four major developmental stages. J Affect Disord. 2018;228:248-253. doi:10.1016/j. jad.2017.12.014

7. Gaynes BN, Magruder KM, Burns BJ, Wagner HR, Yarnall KS, Broadhead WE. Does a coexisting anxiety disorder predict persistence of depressive illness in primary care patients with major depression? Gen Hosp Psychiatry. 1999;21(3):158-167. doi:10.1016/S0163-8343(99)00005-5

8. Penninx BW, Nolen WA, Lamers F, et al. Two-year course of depressive and anxiety disorders: results from the Netherlands study of depression and anxiety (NESDA). J Affect Disord. 2011;133(1-2):76-85. doi:10.1016/j.jad.2011.03.027

9. Otto MW, Simon NM, Wisniewski SR, et al. Prospective 12-month course of bipolar disorder in out-patients with and without comorbid anxiety disorders. Br J Psychiatry. 2006;189(1):20-25. doi:10.1192/ bjp.bp. 104.007773

10. Hawton K, Casanas ICC, Haw C, Saunders K. Risk factors for suicide in individuals with depression: a systematic review. J Affect Disord. 2013;147(1-3):17-28. doi:10.1016/j.jad.2013.01.004 
11. Schaffer A, Isometsa ET, Tondo L, et al. International society for bipolar disorders task force on suicide: meta-analyses and meta-regression of correlates of suicide attempts and suicide deaths in bipolar disorder. Bipolar Disord. 2015;17(1):1-16. doi:10.1111/ bdi. 12271

12. Buhlmann U, Deckersbach T, Engelhard I, et al. Cognitive retraining for organizational impairment in obsessive-compulsive disorder Psychiatry Res. 2006;144(2-3):109-116. doi:10.1016/j. psychres.2005.10.012

13. Burdick KE, Ketter TA, Goldberg JF, Calabrese JR. Assessing cognitive function in bipolar disorder: challenges and recommendations for clinical trial design. J Clin Psychiatry. 2015;76(3):e342-350. doi:10.4088/JCP.14cs09399

14. de Filippis R, Aloi M, Bruni A, Gaetano R, Segura-Garcia C, De Fazio P. Bipolar disorder and obsessive compulsive disorder: the comorbidity does not further impair the neurocognitive profile. $J$ Affect Disord. 2018;235:1-6. doi:10.1016/j.jad.2018.03.010

15. Souery D, Oswald P, Massat I, et al. Clinical factors associated with treatment resistance in major depressive disorder: results from a European multicenter study. $J$ Clin Psychiatry. 2007;68 (7):1062-1070. doi:10.4088/JCP.v68n0713

16. Barbuti M, Pacchiarotti I, Vieta E, et al. Antidepressant-induced hypomania/mania in patients with major depression: evidence from the BRIDGE-II-MIX study. J Affect Disord. 2017;219:187-192. doi:10.1016/j.jad.2017.05.035

17. Masi G, Toni C, Perugi G, Mucci M, Millepiedi S, Akiskal HS. Anxiety disorders in children and adolescents with bipolar disorder: a neglected comorbidity. Can J Psychiatry. 2001;46(9):797-802. doi:10.1177/070674370104600902

18. Goodwin GM, Haddad PM, Ferrier IN, et al. Evidence-based guidelines for treating bipolar disorder: revised third edition recommendations from the British association for psychopharmacology. J Psychopharmacol. 2016;30(6):495-553. doi:10.1177/0269881116636545

19. Zimmerman M, Chelminski I. Clinician recognition of anxiety disorders in depressed outpatients. $J$ Psychiatr Res. 2003;37 (4):325-333. doi:10.1016/S0022-3956(03)00020-7

20. Dineen Wagner K. Bipolar disorder and comorbid anxiety disorders in children and adolescents. J Clin Psychiatry. 2006;67(Suppl 1):16-20.

21. de Graaf R, Bijl RV, Smit F, Vollebergh WA, Spijker J. Risk factors for 12-month comorbidity of mood, anxiety, and substance use disorders: findings from the Netherlands mental health survey and incidence study. Am $J$ Psychiatry. 2002;159(4):620-629. doi:10.1176/appi.ajp.159.4.620

22. Paholpak S, Kongsakon R, Pattanakumjorn W, Kanokvut R, Wongsuriyadech W, Srisurapanont M. Risk factors for an anxiety disorder comorbidity among Thai patients with bipolar disorder: results from the Thai bipolar disorder registry. Neuropsychiatr Dis Treat. 2014;10:803-810. doi:10.2147/NDT.S57019

23. Saunders EF, Fitzgerald KD, Zhang P, McInnis MG. Clinical features of bipolar disorder comorbid with anxiety disorders differ between men and women. Depress Anxiety. 2012;29(8):739-746. doi:10.1002/da.21932

24. Inoue T, Inagaki Y, Kimura T, Shirakawa O. Prevalence and predictors of bipolar disorders in patients with a major depressive episode: the Japanese epidemiological trial with latest measure of bipolar disorder (JET-LMBP). J Affect Disord. 2015;174:535-541. doi:10.1016/j.jad.2014.12.023

25. Otsubo T, Tanaka K, Koda R, et al. Reliability and validity of Japanese version of the mini-international neuropsychiatric interview. Psychiatry Clin Neurosci. 2005;59(5):517-526. doi:10.1111/j.1440-1819.2005.01408.x

26. Sheehan DV, Lecrubier Y, Sheehan KH, et al. The mini-international neuropsychiatric interview (M.I.N.I.): the development and validation of a structured diagnostic psychiatric interview for DSM-IV and ICD-10. J Clin Psychiatry. 1998;59(Suppl 20):22-33.
27. Fujisawa D, Nakagawa A, Tajima M, et al. Cross-cultural adaptation of the quick inventory of depressive symptomatology-self report (QIDS-SR-J). Jap J Stress Sci. 2010;25(1):43-52.

28. Fukuhara S, Bito S, Green J, Hsiao A, Kurokawa K. Translation, adaptation, and validation of the SF-36 health survey for use in Japan. J Clin Epidemiol. 1998;51(11):1037-1044. doi:10.1016/ S0895-4356(98)00095-X

29. Nakai Y, Inoue T, Toda H, et al. The influence of childhood abuse, adult stressful life events and temperaments on depressive symptoms in the nonclinical general adult population. $J$ Affect Disord. 2014;158:101-107. doi:10.1016/j.jad.2014.02.004

30. Sanders B, Becker-Lausen E. The measurement of psychological maltreatment: early data on the child abuse and trauma scale. Child Abuse Negl. 1995;19(3):315-323. doi:10.1016/S0145-2134(94)00131-6

31. Tanabe H, Goto K, Ozawa S. Psychometric properties of the Japanese version of the child abuse and trauma scale (CATS) [abstract]. In: The 9th Annual Meeting of the Japanese Society for Traumatic Stress Studies; Mar 6, 2010; Kobe, Japan.

32. Howland RH, Rush AJ, Wisniewski SR, et al. Concurrent anxiety and substance use disorders among outpatients with major depression: clinical features and effect on treatment outcome. Drug Alcohol Depend. 2009;99 (1-3):248-260. doi:10.1016/j.drugalcdep.2008.08.010

33. Simon NM, Otto MW, Wisniewski SR, et al. Anxiety disorder comorbidity in bipolar disorder patients: data from the first 500 participants in the systematic treatment enhancement program for bipolar disorder (STEP-BD). Am J Psychiatry. 2004;161 (12):2222-2229. doi:10.1176/appi.ajp.161.12.2222

34. Yapici Eser H, Kacar AS, Kilciksiz CM, Yalçinay-Inan M, Ongur D. Prevalence and associated features of anxiety disorder comorbidity in bipolar disorder: a meta-analysis and meta-regression study. Front Psychiatry. 2018;9:229. doi:10.3389/fpsyt.2018.00229

35. Pavlova B, Perroud N, Cordera P, Uher R, Dayer A, Aubry JM. Childhood maltreatment and comorbid anxiety in people with bipolar disorder. $J$ Affect Disord. 2016;192:22-27. doi:10.1016/j. jad.2015.12.002

36. Agnew-Blais J, Danese A. Childhood maltreatment and unfavourable clinical outcomes in bipolar disorder: a systematic review and meta-analysis. Lancet Psychiatry. 2016;3(4):342-349. doi:10.1016/ S2215-0366(15)00544-1

37. Lamers F, van Oppen P, Comijs HC, et al. Comorbidity patterns of anxiety and depressive disorders in a large cohort study: the Netherlands study of depression and anxiety (NESDA). J Clin Psychiatry. 2011;72(3):341-348. doi:10.4088/JCP.10m06176blu

38. Hovens JG, Giltay EJ, Spinhoven P, van Hemert AM, Penninx BW. Impact of childhood life events and childhood trauma on the onset and recurrence of depressive and anxiety disorders. J Clin Psychiatry. 2015;76(7):931-938. doi:10.4088/JCP.14m09135

39. Cyranowski JM, Schott LL, Kravitz HM, et al. Psychosocial features associated with lifetime comorbidity of major depression and anxiety disorders among a community sample of mid-life women: the SWAN mental health study. Depress Anxiety. 2012;29(12):1050-1057. doi:10.1002/da.21990

40. Brown GR, McBride L, Bauer MS, Williford WO. Cooperative studies program 430 study T. Impact of childhood abuse on the course of bipolar disorder: a replication study in U.S. veterans. J Affect Disord. 2005;89(1-3):57-67. doi:10.1016/j.jad.2005.06.012

41. Chen LP, Murad MH, Paras ML, et al. Sexual abuse and lifetime diagnosis of psychiatric disorders: systematic review and meta-analysis. Mayo Clin Proc. 2010;85(7):618-629. doi:10.4065/ mcp.2009.0583

42. Matza LS, Revicki DA, Davidson JR, Stewart JW. Depression with atypical features in the National comorbidity survey: classification, description, and consequences. Arch Gen Psychiatry. 2003;60 (8):817-826. doi:10.1001/archpsyc.60.8.817 
43. Posternak MA, Zimmerman M. The prevalence of atypical features across mood, anxiety, and personality disorders. Compr Psychiatry. 2002;43(4):253-262. doi:10.1053/comp.2002.33498

44. Benazzi F. Exploring aspects of DSM-IV interpersonal sensitivity in bipolar II. J Affect Disord. 2000;60(1):43-46. doi:10.1016/S01650327(99)00174-3

45. Gao S, Assink M, Cipriani A, Lin K. Associations between rejection sensitivity and mental health outcomes: a meta-analytic review. Clin Psychol Rev. 2017;57:59-74. doi:10.1016/j.cpr.2017.08.007

46. Meng X, D'Arcy C. Common and unique risk factors and comorbidity for 12-month mood and anxiety disorders among Canadians. Can J Psychiatry. 2012;57(8):479-487. doi:10.1177/070674371205700806

47. Scott KM, Wells JE, Angermeyer M, et al. Gender and the relationship between marital status and first onset of mood, anxiety and substance use disorders. Psychol Med. 2010;40(9):1495-1505. doi:10.1017/S0033291709991942

48. Andrade L, Eaton WW, Chilcoat H. Lifetime comorbidity of panic attacks and major depression in a population-based study. Symptom profiles. $B r \quad J$ Psychiatry. 1994;165(3):363-369. doi:10.1192/ bjp. 165.3 .363
49. Gyulai L, Bauer MS, Marangell LB, et al. Correlates of functioning in bipolar disorder. Psychopharmacol Bull. 2008;41(4):51-64.

50. Rapaport MH, Clary C, Fayyad R, Endicott J. Quality-of-life impairment in depressive and anxiety disorders. Am J Psychiatry. 2005;162 (6):1171-1178. doi:10.1176/appi.ajp.162.6.1171

51. Sylvia LG, Montana RE, Deckersbach T, et al. Poor quality of life and functioning in bipolar disorder. Int $J$ Bipolar Disord. 2017;5 (1):10. doi:10.1186/s40345-017-0078-4

52. Bauer MS, Altshuler L, Evans DR, Beresford T, Williford WO, Hauger R. Prevalence and distinct correlates of anxiety, substance, and combined comorbidity in a multi-site public sector sample with bipolar disorder. J Affect Disord. 2005;85(3):301-315. doi:10.1016/j. jad.2004.11.009

53. Lin CH, Wang FC, Lin SC, Chen CC, Huang CJ. A comparison of inpatients with anxious depression to those with nonanxious depression. Psychiatry Res. 2014;220(3):855-860. doi:10.1016/j. psychres.2014.08.048

\section{Publish your work in this journal}

Neuropsychiatric Disease and Treatment is an international, peerreviewed journal of clinical therapeutics and pharmacology focusing on concise rapid reporting of clinical or pre-clinical studies on a range of neuropsychiatric and neurological disorders. This journal is indexed on PubMed Central, the 'PsycINFO' database and CAS, and is the official journal of The International Neuropsychiatric Association (INA). The manuscript management system is completely online and includes a very quick and fair peer-review system, which is all easy to use. Visit http://www.dovepress.com/testimonials.php to read real quotes from published authors. 